\author{
Ecología
}

\title{
Patrones de distribución espacial del arbolado en un bosque mixto de pino-encino del noreste de México
}

\author{
Spatial distribution patterns of trees in a mixed pine-oak forest of Northeastern Mexico \\ Ernesto Alonso Rubio-Camacho ${ }^{\mathrm{a}}$, Marco Aurelio González-Tagle ${ }^{\mathrm{b}, *}$, Wibke Himmelsbach ${ }^{\mathrm{b}}$, \\ Diana Yemilet Ávila-Flores ${ }^{\mathrm{c}}$, Eduardo Alanís-Rodríguez ${ }^{\mathrm{b}}$ y Javier Jiménez-Pérez ${ }^{\mathrm{b}}$ \\ a INIFAP-CIRPAC, C. E. Centro Altos de Jalisco, Carretera libre Tepatitlán-Lagos de Moreno, Apartado postal 56, 47600, Tepatitlán, Jalisco, México \\ ${ }^{\mathrm{b}}$ Facultad de Ciencias Forestales, Universidad Autónoma de Nuevo León, Carretera Nacional km 147, 67700, Linares, Nuevo León, México \\ c INIFAP-CIRNE, C. E. Saltillo, Carretera Saltillo-Zacatecas km 342+19, Hacienda de Buena Vista, 25315, Saltillo, Coahuila, México
}

Recibido el 18 de febrero de 2015; aceptado el 11 de octubre de 2015

Disponible en Internet el 23 de febrero de 2017

\begin{abstract}
Resumen
En este estudio se aborda la caracterización de los patrones de distribución espacial del arbolado en un bosque mixto del noreste de México. Se empleó un análisis de patrón de puntos para evaluar mezcla de especies, diferenciación dimensional y patrones de agregación. Los datos se obtuvieron de 2 parcelas de una hectárea cada una. Se registraron azimut y distancia para cada árbol, especie, altura y diámetro. La información se analizó por parcela y por grupos, correspondientes a los géneros más abundantes (Pinus, Quercus y Juniperus). Los resultados indican una distribución aleatoria con la prueba de uniformidad de ángulos (Wi), en general y para ambas parcelas. El grado de mezcla de especies $(\mathrm{Mi})$ muestra que en promedio la especie referencia tiene vecinos de diferente especie, la diferenciación dimensional (TDi) indica diferencias de moderadas a claras en el análisis general. Las diferencias se manifestaron en la dominancia dimensional (UDi) por género para ambas parcelas, lo cual evidenció la influencia de un régimen local de incendios. El análisis de la estructura y dinámica espacial en bosques mixtos, mediante análisis generalizados y específicos, brinda un panorama detallado sobre el comportamiento del bosque, cuyos resultados se pueden asociar al estudio de perturbaciones. (C) 2017 Universidad Nacional Autónoma de México, Instituto de Biología. Este es un artículo Open Access bajo la licencia CC BY-NC-ND (http://creativecommons.org/licenses/by-nc-nd/4.0/).
\end{abstract}

Palabras clave: Patrón de puntos; Estimador NN1; Índice de agregación; Índice de mezcla de especies

\section{Abstract}

The objective of this study was to characterize the tree spatial structure patterns of the mixed pine-oak forest in the northeast part of Mexico. A point pattern analysis was conducted in order to evaluate the following indices: aggregation index, mingling species index, dimensional (dbh and height) index and dominance index. The data were collected from 2 stands of 1 ha each. The variables obtained for all trees were: Azimuth and distance of each tree to the center of the stand, $\mathrm{DBH}$, species, and height. The data were analyzed for each stand and also as a groups corresponding to the most abundant genera in the stands: (Pinus, Quercus, and Juniperus). The results show that according to the aggregation index ( $W_{i}$ ) both stands presents a random distribution, the mingling species $\left(M_{i}\right)$ shows a higher mixture at both stands and the dimensional index shows moderate differences in DBH and height in both stands. The main differences were found using the dominance index $\left(U_{i}\right)$ based on the groups; by this means a particular and local fire regime was identified for the area. The use of spatial analysis and the quantification of stand structure help to depict a detailed status of the forest and also the effects of disturbance on the forests.

(C) 2017 Universidad Nacional Autónoma de México, Instituto de Biología. This is an open access article under the CC BY-NC-ND license (http://creativecommons.org/licenses/by-nc-nd/4.0/).

Keywords: Point pattern; Estimate NN1; Aggregation index; Mingling index

\footnotetext{
* Autor para correspondencia.

Correoelectrónico: marco.gonzaleztg@uanl.edu.mx (M.A. González-Tagle).

La revisión por pares es responsabilidad de la Universidad Nacional Autónoma de México.
} 


\section{Introducción}

Los ecosistemas forestales mexicanos además de proveer múltiples bienes y servicios se encuentran expuestos a una gran variedad de desastres naturales y procesos antropogénicos. De esta manera, después de la presencia de un incendio o actividades silvícolas, los bosques experimentan cambios inmediatos en los patrones de distribución del arbolado y en la variación de las características de la estructura forestal que modifican el grado de competencia, crecimiento y regeneración del bosque (Ávila et al., 2014, Balvanera et al., 2009, Gadow et al., 2012, Gadow, Sánchez y Álvarez, 2007; Merganič, Merganičová, Marušák y Audolenská, 2012). Sin embargo, a pesar de su extrema importancia aún existe una escasa disponibilidad de datos tanto cualitativos como cuantitativos que ofrezcan información detallada sobre la diversidad de la estructura y los patrones de distribución espacial del arbolado.

De modo que la diversidad de la estructura espacial se puede caracterizar utilizando 3 factores: la agregación espacial, el grado de mezcla de las especies y el patrón de diferenciación espacial por dimensiones (Gadow et al., 2007; Pommerening, 2002). Estas 3 características se pueden obtener mediante índices de patrón de puntos y relaciones de vecindad (Nearest Neighbor Summary Statistics), y constituyen un elemento importante para el estudio de la dinámica de los ecosistemas así como del régimen de las perturbaciones a las que se encuentren sometidos dichos ecosistemas (Ávila et al., 2012; Gadow et al., 2012; González-Tagle, Schwendemann, Jiménez y Schulz, 2008; Pommerening, 2008).

Para el presente estudio se plantea la siguiente hipótesis: Las características de la estructura y diversidad espacial son diferentes en 2 rodales con distinto tiempo de intervalo de retorno de los incendios. En este sentido, se investigó mediante las relaciones de vecindad el grado en que diferentes frecuencias de incendios de baja severidad modifica las siguientes características de un rodal: 1) la distribución espacial, 2) el grado de mezcla de las especies y 3) el patrón de diferenciación espacial por dimensiones en un bosque mixto de pino-encino en la sierra Madre Oriental de México.

Conocer las particularidades de los efectos de la frecuencia de incendios sobre los ecosistemas boscosos de México no solo permitirá orientar de manera adecuada una gestión sustentable de los recursos forestales, sino que, a la par, se podrán establecer acciones dirigidas a la restauración de los ecosistemas afectados por los incendios forestales (González-Tagle et al., 2008).

\section{Materiales y métodos}

El trabajo de investigación se llevó a cabo en el área del Campus Ecológico Iturbide, manejado por la Universidad Autónoma de Nuevo León. El Campus Ecológico Iturbide se encuentra ubicado a $15 \mathrm{~km}$ al sureste del municipio de Iturbide en el estado de Nuevo León, México, entre las coordenadas $24^{\circ} 42^{\prime} \mathrm{N}$ y los $99^{\circ} 51^{\prime} \mathrm{O}$ y cubre una superficie aproximada de 1,035 ha, con un rango altitudinal de 1,200-1,890 m snm (Himmelsbach et al., 2011). El área de muestreo se ubicó en la zona del bosque de pino-encino, que por sus características bioclimáticas y físicas se clasifica como un ecosistema con régimen de incendios frecuentes y de baja severidad (Jardel-Peláez, Alvarado-Celestino, Morfín-Ríos, Castillo-Navarro y FloresGarnica, 2009; Rodríguez, 2008). En esta área se seleccionaron 2 rodales distintos (fig. 1) con base en las evidencias de incendios detectadas en un estudio anterior por Rubio-Camacho, González, Alanís, Chávez y Aguirre (2015). En el rodal P1 se encontraron evidencias de incendio tales como árboles con la corteza carbonizada y menor carga de combustible $(36.6 \mathrm{Mg} / \mathrm{ha})$ sobre todo en la capa orgánica del suelo, características totalmente opuestas en el rodal P2, es decir, sin presencia de cicatrices de incendios y una carga de combustible significativamente mayor ( $<<0.001$ ) de $49.6 \mathrm{Mg} / \mathrm{ha}$ (Rubio-Camacho, González, Benavides, Chávez y Xelhuantzi, 2016). De acuerdo con Agee (1993) y Whelan (1995), las evidencias en el rodal P1 son resultado de un intervalo de retorno más corto, por lo cual, para fines del presente estudio, se asume que existen 2 frecuencias distintas y se toma como un elemento válido de comparación entre los rodales (intervalo menor el rodal P1 vs. intervalo mayor el rodal P2).

Los géneros más importantes en los rodales de estudio son Pinus, Quercus y Juniperus (Rubio-Camacho et al., 2015), cada uno con diferentes adaptaciones al fuego. El género Pinus, específicamente P. pseudostrobus, es intolerante a la sombra, también presenta una corteza gruesa asociada a la resistencia a los incendios y se ha observado regeneración de copa después de que han sido quemados, sobre todo en arbolado joven (Rodríguez y Fulé, 2003). Para el caso de Quercus se sabe que algunas especies tienden a rebrotar tras el paso de los incendios y que son tolerantes a la sombra (González-Tagle et al., 2008; Rodríguez y Myers, 2010). Aunque para Juniperus flaccida no se encontró información sobre su ecología del fuego, se sabe que otras especies del género son altamente vulnerables a los incendios, además, tiene alto contenido de aceites volátiles y una corteza que se desprende del tronco, y estas características la hacen altamente vulnerable a los incendios (Sullivan, 2007).

El muestreo del arbolado se realizó mediante el establecimiento de una parcela de 1 ha $(100 \times 100 \mathrm{~m})$ para cada rodal, las cuales se denominan como P1 y P2. Estas parcelas se dividieron en subparcelas de $20 \times 20 \mathrm{~m}\left(400 \mathrm{~m}^{2}\right)$, quedando un total de 25 subparcelas en cada parcela. De esta manera, se realizó un inventario de todos los árboles dentro de cada subparcela, donde se registró el diámetro del tronco a la altura de $1.30 \mathrm{~m}$ $\left(\mathrm{d}_{1.3}\right)$, altura total y se determinó la especie. A cada uno de los árboles registrados se les tomó la distancia y azimut a partir del centro de la subparcela.

Para la caracterización de la diversidad espacial del arbolado en la zona de estudio se utilizaron técnicas de análisis de patrón de puntos y relaciones de vecindad. Con excepción del índice de Clark y Evans (1954), todos los demás están basados en el método de los 5 árboles (Albert, 1999; Hui y Hu, 2001) el cual toma un árbol de referencia (i) y sus 4 vecinos más cercanos. Los gráficos, análisis y la conformación de los grupos estructurales se realizaron mediante el programa $\mathrm{R} v 2.15$, con apoyo en los scripts proporcionados por el Woodland Structure Analysis and Modelling Lab. (Pommerening, 2013) y con soporte de las 


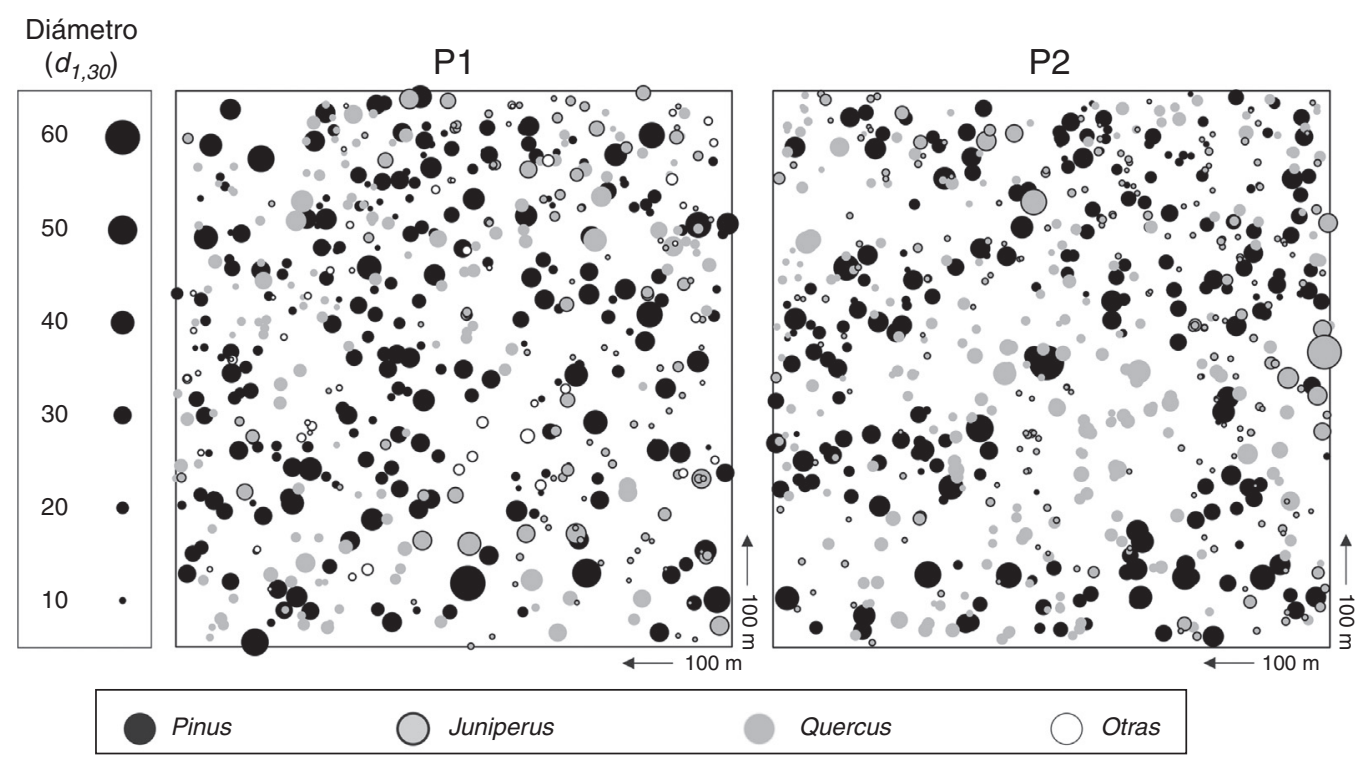

Figura 1. Representación de la distribución espacial del arbolado por parcela en el área de estudio. Los círculos representan los árboles, cuyo tamaño está escalado al diámetro normal y los diferentes colores representan a los grupos estudiados. P1: parcela 1; P2: parcela 2. Pinus, Quercus y Juniperus son los grupos de estudio.

librerías SPATSTAT (Baddeley y Turner, 2005) y LATTICE (Sarkar, 2013).

\section{Patrones de agregación del arbolado}

El índice de agregación de Clark y Evans es una medida de agrupamiento u ordenamiento de un patrón de puntos (Aguirre, 2004; Pommerening, 2002). Describe la relación de la distancia media observada ( $\bar{r}$ observada) del vecino más cercano con respecto a la esperada ( $\bar{r}$ esperada) en una distribución de puntos tipo Poisson o aleatoria.

$\mathrm{R}=\frac{\overline{\mathrm{r}} \text { observada }}{\overline{\mathrm{r}} \text { esperada }}$

Teóricamente, $R$ puede tomar valores entre 0 (mayor grado de agrupamiento) y 2.1491 (patrón regular de forma hexagonal), e indica cuán regular, aleatoria o agrupada es la distribución espacial del arbolado en un área determinada. Valores de agregación menores a 1 , cercanos a 1 y mayores a 1 indican agrupamiento, aleatoriedad y distribución regular, respectivamente (Aguirre, 2004; Pommerening, 2002).

$R$ es determinada mediante análisis de vecindad con respecto al vecino más cercano. Para lo cual se mide la distancia de cada árbol a su vecino más cercano en un área determinada, la distancia media de cada árbol se calcula como sigue:

$\bar{r}$ observada $=\frac{\sum_{i=1}^{n} r_{i}}{n}$

en tanto la $\overline{\mathrm{r}}$ esperada se calcula de la siguiente manera:

$\bar{r}$ esperada $=\frac{1}{2 \sqrt{N / A}}$

donde $A=$ área de la parcela y $N=$ número de árboles en la parcela.

El índice de uniformidad de Gadow ( $W_{i}$, Gadow et al., 1998, Gado, Hui y Albert, 1998) representa una alternativa al índice de agregación y describe la regularidad de la distribución del arbolado (Aguirre, 2004; Gadow et al., 2007; Pommerening, 2002). Para su determinación es necesario conocer el ángulo entre 2 árboles vecinos con respecto al árbol cero, el cual se compara con un ángulo de referencia $\alpha_{0}$, y se obtiene de la siguiente fórmula:

$\alpha_{0}=360 / n$

donde $n=$ número de árboles vecinos considerados.

Para el cálculo del índice $W_{i}$ se define la proporción de ángulos menores al ángulo de referencia $\alpha_{0}$ :

$W_{i}=\frac{1}{n} \sum_{j=1}^{n} w_{i j}$

donde $w_{\mathrm{ij}}=1$, si el ángulo ${ }_{j}$ es menor al $\alpha_{0}, w_{\mathrm{ij}}=0$, si es mayor. En un grupo estructural de 4 árboles Wi puede obtenerse comparando el ángulo de referencia $90^{\circ}$ y puede presentar valores de $0,0.25,0.50,0.75$ y 1.00 , cero cuando ninguno de los ángulos es menor a $\alpha_{0}$ y 1 cuando todos son menores a $\alpha_{0}$.

El promedio del índice de uniformidad $\bar{W}_{i}$ puede utilizarse para clasificar los rodales en categorías de distribución espacial agregada, regular y aleatoria. Valores de $\bar{W}_{i}$ mayores que 0.6 se pueden considerar como patrón de agregación, valores entre 0 y 0.4 indican una distribución regular y aquellos valores medios entre 0.4 y 0.6 indican una distribución aleatoria (Aguirre, 2004; Gadow et al., 2007; Pommerening, 2002).

\section{Grado de mezcla en la distribución espacial de especies}

Índice de mezcla de especies $\left(M_{i}\right)$. Una medida de la diversidad en la distribución espacial de especies es el grado de mezcla entre estas, el cual se puede estimar mediante el índice $M_{i}$. El índice de mezcla $M_{i}$ de Füldner (1995) se define como la proporción de $n$ vecinos más cercanos que no pertenecen a la misma 
especie que el árbol de referencia, en este caso se toman los 4 vecinos más cercanos.

$M_{i}=\frac{1}{n} \sum_{j=1}^{n} v_{j}$

donde $0 \leq M_{i} \leq 1, v_{j}$ es igual a 0 cuando el árbol $j$ es de la misma especie que el árbol de referencia $i$ y será 1 en caso contrario (Gadow et al., 2007).

El grado de diferenciación dimensional constituye una forma de describir la proximidad espacial que existe entre árboles de diferentes dimensiones en el bosque. Una forma de medir esta diferenciación es con los índices denominados grado de diferenciación, y dominancia dimensional (Gadow et al., 2007).

El índice de diferenciación dimensional $\left(T_{i}\right)$ constituye un índice que se puede aplicar a cualquier variable que represente el tamaño del árbol, en este caso a diámetros y alturas. Toma en cuenta los $n$ árboles más cercanos al árbol de referencia $i$ y se define de la siguiente manera:

$T_{i}=1-\frac{1}{n} \sum_{j=1}^{n} \frac{\min \left(\operatorname{Dim}_{i}, \operatorname{Dim}_{j}\right)}{\max \left(\operatorname{Dim}_{i}, \operatorname{Dim}_{j}\right)}$

donde $T_{i}=$ grado de diferenciación en diámetros o/y alturas, $j=1 \ldots n$ árboles vecinos, $i=$ árbol de referencia, $\operatorname{Dim}_{i}=$ diámetro o altura del árbol $\boldsymbol{i}, \operatorname{Dim}_{j}=$ diámetro o altura del árbol $j$.

El valor del $T_{i}$ se incrementa al aumentar la diferencia media del tamaño de los árboles vecinos $\left(0 \leq T_{i} \leq 1\right)$. Entre más bajo sea el valor de $T_{i}$ las diferencias son menores, un valor de 0 indica que todos los árboles son del mismo tamaño (Gadow et al., 2007). Siguiendo el método de Aguirre, Kramer y Jiménez (1998), y Jiménez, Aguirre y Kramer (1998), el grado de diferenciación en diámetros y alturas se clasificó en 5 categorías, de 0-0.2 = débil; 0.2-0.4 = moderada; 0.4-0.6 clara; 0.6-0.8 fuerte; 0.8-1.0 muy fuerte.

Otro índice para la diferenciación dimensional es la medida de entorno o índice de dominancia $\left(U_{i}\right)$. Se define como la dominancia del árbol referencia $i$ con respecto a sus vecinos más cercanos, es decir, la proporción de árboles $n$ más pequeños (en diámetro o altura) que el árbol de referencia.

$U_{i}=\frac{1}{n} \sum_{j=1}^{n} v_{j}$

donde $0 \leq U_{i} \leq 1$ y $v_{j}$ será igual a 1 si el árbol $j$ es menor al árbol referencia $i$, y 0 en caso contrario. Con 4 vecinos el índice de dominancia $U_{i}$ puede tomar 5 valores y es muy útil cuando se desea ver la dominancia relativa de una especie en particular (Aguirre, Hui, von Gadow y Jiménez, 2003; Gadow et al., 2007).

Estos índices se calculan dentro de una ventana de observación $(W)$, en este caso son los polígonos del área de las parcelas P1 y P2. Para cada una de estas se realiza el análisis de la información con las fórmulas anteriormente descritas, pero se debe tener en consideración el efecto de borde de la ventana (Pommerening y Stoyan, 2006).

El efecto de borde desempeña un papel importante para la correcta caracterización de la estructura espacial. Los árboles que se encuentran cerca del borde $W$ suelen tener parte de sus vecinos más cercanos del otro lado, es decir, fuera de la parcela, lo que implica un error en la estimación de los valores de vecindad de los árboles que se encuentran dentro. Para evitar esto se han propuesto varios métodos descritos y probados por Pommerening y Stoyan (2006), entre ellos se encuentra el estimador NN1 el cual se utilizó en este estudio.

El estimador NN1 consiste en omitir como árbol de referencia (i) a aquellos cuya distancia a su j-ésimo vecino más cercano sea menor a la distancia entre este y el borde de $W$, no obstante, esto no los omite de formar parte de otros grupos estructurales como vecino más cercano. Para más detalles consultar Pommerening y Stoyan (2006).

Para el cálculo de la prueba estadística del índice de Clark y Evans se utilizaron 999 permutaciones de Monte Carlo para probar la distribución completamente aleatoria (CSR, por sus siglas en inglés con un alfa $=0.05$ ). Esto es, para comprobar si el índice resultante es estadísticamente diferente o igual a 1 con un $95 \%$ de confianza (Baddeley y Turner, 2005).

Además, se llevaron a cabo contrastes estadísticos entre parcelas, tomado como factor la diferencia en cargas de necromasa entre ambas. Esto se llevó a cabo mediante procedimientos de remuestreo estadístico, específicamente se implementó la paquetería «WRS2» en el programa $\mathrm{R}$, mediante la cual se usaron simulaciones bootstrap para calcular la diferencia entre las medias con un IC del 95\% (Mair, Wilcox y Schoenbrodt, 2015; Wilcox, 2005).

\section{Resultados}

Los resultados se presentan por parcela (incluyen a todos los individuos) y con base en grupos de acuerdo con los géneros Pinus, Quercus y Juniperus, por ser los más abundantes y con mayores dimensiones (tabla 1). En la figura 1 se observa la distribución espacial del arbolado por grupo en las parcelas P1 y P2.

Con la aplicación del estimador NN1 (Pommerening y Stoyan, 2006) se omitieron aquellos árboles afectados por el efecto de borde $(W)$ y los grupos quedaron como se describe a continuación. Originalmente en la P1 se registraron 561 grupos, de los cuales, al aplicar la corrección $W$ se utilizaron 485 grupos estructurales de los 5 árboles; para la P2 se contó con un total de 669 grupos y solo quedaron 561 grupos. Para el análisis de grupos, en la P1 Pinus tuvo 213 grupos estructurales, Quercus 129 y Juniperus 100, para la P2 en el grupo de Pinus se contabilizaron 193 grupos estructurales, en Quercus 189 grupos y en el grupo Juniperus 87 grupos.

\section{Patrones de agregación del arbolado}

Índice de agregación de Clark y Evans (R). Para el índice R se obtuvieron valores similares en ambas parcelas, $0.93(p=0.002)$ para la P1 y $0.91(p=0.002)$ para la P2. Estos valores representan una distribución agregada de los árboles cuando se incluyen todas las especies. Por otro lado, el análisis por grupos indica que todos ellos tienden a una distribución agrupada. El grupo Pinus en la P1 muestra un valor cercano a la aleatoriedad (0.94, 
Tabla 1

Características dasométricas de las parcelas P1 y P2 del área de estudio.

\begin{tabular}{|c|c|c|c|c|c|c|c|c|}
\hline \multirow[b]{2}{*}{ Especie } & \multicolumn{4}{|c|}{ Parcela 1 (P1) } & \multicolumn{4}{|c|}{ Parcela 2 (P2) } \\
\hline & $\mathrm{Nha}^{-1}$ & $\begin{array}{l}\mathrm{G} \\
\left(\mathrm{m}^{2} \cdot \mathrm{ha}^{-1}\right)\end{array}$ & $d_{1.30}(\mathrm{~cm})$ & $\mathrm{AT}(\mathrm{m})$ & $\mathrm{Nha}^{-1}$ & $\begin{array}{l}\mathrm{G} \\
\left(\mathrm{m}^{2} \cdot \mathrm{ha}^{-1}\right)\end{array}$ & $d_{1.30}(\mathrm{~cm})$ & $\mathrm{AT}(\mathrm{m})$ \\
\hline Pinus pseudostrobus & 242 & 12.2282 & 23.1 & 11.8 & 221 & 11.7225 & 24.2 & 12.2 \\
\hline Juniperus flaccida & 116 & 2.6832 & 15.4 & 7.5 & 109 & 2.3077 & 14.1 & 7.6 \\
\hline Quercu canbyi & 82 & 1.9539 & 16.2 & 6.9 & 213 & 5.1425 & 16.6 & 7.3 \\
\hline Quercus rysophylla & 43 & 1.2664 & 17.9 & 7.1 & 19 & 0.4887 & 16 & 6.6 \\
\hline Arbutus xalapensis & 31 & 0.4852 & 13.4 & 4.7 & 33 & 0.3262 & 10.9 & 4.9 \\
\hline Quercus polymorpha & 22 & 0.6007 & 17.8 & 5.8 & 4 & 0.1196 & 19.4 & 8.9 \\
\hline Carya myristiciformis & 12 & 0.1423 & 11.7 & 6.6 & - & - & - & - \\
\hline Mimosa texana & 4 & 0.0321 & 9.7 & 4.6 & - & - & - & - \\
\hline Prunus serotina & 4 & 0.0615 & 13.4 & 5.6 & - & - & - & - \\
\hline Rhus virens & 1 & 0.0119 & 12.3 & 3.7 & 68 & 0.5762 & 10.1 & 3.8 \\
\hline Acacia coulteri & 1 & 0.0100 & 11.3 & 4.6 & - & - & - & - \\
\hline Malacomeles denticulata & 1 & 0.0049 & 7.9 & 3.4 & - & - & - & - \\
\hline Prosopis laevigata & 1 & 0.0095 & 11 & 6.1 & - & - & - & - \\
\hline Quercus laceyi & 1 & 0.0181 & 15.2 & 5 & - & - & - & - \\
\hline Cercis canadensis & - & - & - & - & 1 & 0.0058 & 8.6 & 5.1 \\
\hline Acacia farnesiana & - & - & - & - & 1 & 0.0059 & 8.7 & 3.3 \\
\hline
\end{tabular}

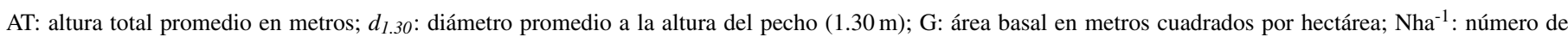
árboles por hectárea.

$p=0.102)$, sin embargo, no significativo; mientras que para P2 presenta una distribución agregada con $0.89(p<0.01)$. Para el grupo Quercus se encontró que en ambas parcelas (P1 y P2) se agrupan con valores de 0.79 y $0.86(p=0.002)$, respectivamente; de igual manera se presenta para el grupo Juniperus, que muestran valores de $0.81(p=0.002)$ en P1 y $0.71(p=0.002)$ en P2.

Sin embargo, al aplicar el análisis de uniformidad de ángulos $\left(W_{i}\right)$ que presenta mayor sensibilidad a los cambios, los resultados muestran que los árboles de manera general tienen una distribución aleatoria. El promedio para las 2 parcelas es de 0.49 $( \pm 0.19)$. En la figura 2 se puede observar que la distribución de las frecuencias por grupos estructurales tiene mayor porcentaje en la categoría 0.5 , lo cual coincide con el promedio general.

El análisis por promedio de cada grupo muestra también tendencia a distribución aleatoria del arbolado sin que se presente una diferencia significativa entre las parcelas. Para el grupo Pinus se tienen promedios de $0.47( \pm 0.18)$ y $0.48( \pm 0.19)$ para P1 y P2, respectivamente; para Quercus con 0.49 ( \pm 18$)$ y 0.50 $( \pm 18)$ y para Juniperus $0.53( \pm 0.19)$ y $0.52( \pm 0.17)$, siendo estos últimos los que presentan una ligera tendencia a la agregación. En la figura 2 se puede observar que la mayor frecuencia de grupos estructurales se encuentra en la categoría de 0.5 , que coindice con el análisis generalizado por parcelas. Esto quiere decir que 2 de los 4 árboles vecinos tienen un ángulo menor a $90^{\circ}$; esta distribución indica que la mayoría de los grupos se encuentran en la categoría de distribución aleatoria.

Índice de mezcla de especies $\left(M_{i}\right)$. El valor promedio en el grado de mezcla para las parcelas P1 y P2 fue de $0.57( \pm 0.28)$ y $0.58( \pm 0.32)$, respectivamente. En la figura 3 se puede observar la distribución de las frecuencias para el índice de $M_{i}$; en la P1 la mayor frecuencia se encuentra en la categoría 0.5 , lo que indica que la mayoría $(>35 \%$ ) de los individuos muestreados está rodeado por al menos 2 individuos de especies diferentes.
Para la P2 se tiene que la mayoría de sus árboles (30\%) están rodeados por 3 por individuos de especies diferentes, lo que indica un mayor grado de mezcla en esta parcela, sin embargo, la comparación estadística no arroja diferencias estadísticamente significativas.

Al realizar el análisis por grupo se encontró que Pinus tiende a mezclarse más en la P2 que en la P1, y para Quercus ocurre lo contrario. Los promedios de $M_{i}$ para Pinus fueron 0.49 $( \pm 27)$ en la P1 y $0.56( \pm 0.29)$ en la P2, cuyas diferencias sí son estadísticamente significativas $(p=0.010)$, con IC entre -0.129 y -0.017 , respectivamente. Quercus también muestra estás diferencias con $0.59( \pm 24)$ y $0.45( \pm 0.34)$ en P1 y P2 ( $p<0.001$; IC $=0.07,0.198)$; en tanto para Juniperus se obtuvo $0.62( \pm 0.29)$ en la P1 y $0.69( \pm 0.24)$ en la P2, para este no se registraron diferencias significativas.

Los gráficos de distribuciones del grado de mezcla $\left(M_{i}\right)$ por grupos concuerdan con los promedios generales (fig. 3). El grupo Pinus tiene su mayor frecuencia en la categoría de 0.50 y en la de 0.75 para P1 y P2, respectivamente, lo que indica que existe un mayor grado de mezcla del género en la P2. En el caso de Quercus, las distribuciones muestran algunas diferencias, en la P1 la categoría que domina es la de 0.50 , en tanto que en la P2 no se observa mucha diferencia en la distribución. Para el caso de Juniperus en la P1, la categoría que presenta mayor proporción es la de 0.50 y para la $\mathrm{P} 2$ la categoría de 0.75 muestra un mayor porcentaje ( $>40)$, indicando un mayor grado de mezcla del género en la P2.

\section{Grado de diferenciación espacial por dimensiones}

Índice de diferenciación dimensional diamétrica $\left(T D_{i}\right)$. Las parcelas de estudio muestran una diferenciación diamétrica entre moderada y clara sin que se presenten diferencias significativas. En la P1 la mayoría de las observaciones (50\%) se encuentran en 


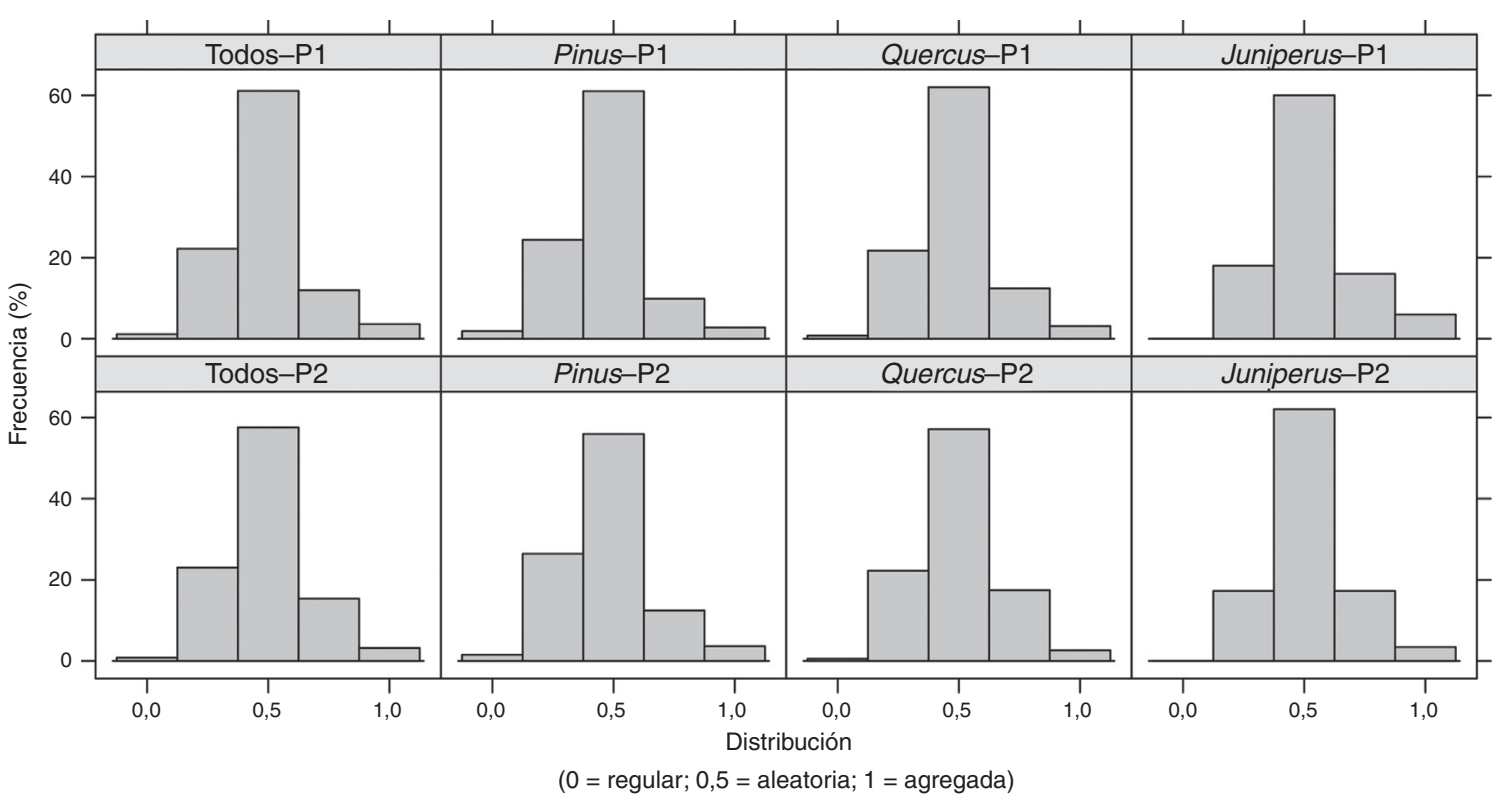

Figura 2. Distribución del índice de uniformidad de ángulos (Wi) por parcela y grupo en el área de estudio. P1: parcela 1; P2: parcela 2. Pinus, Quercus y Juniperus son los grupos de estudio; el grupo Todos incluye a todos los individuos y especies.

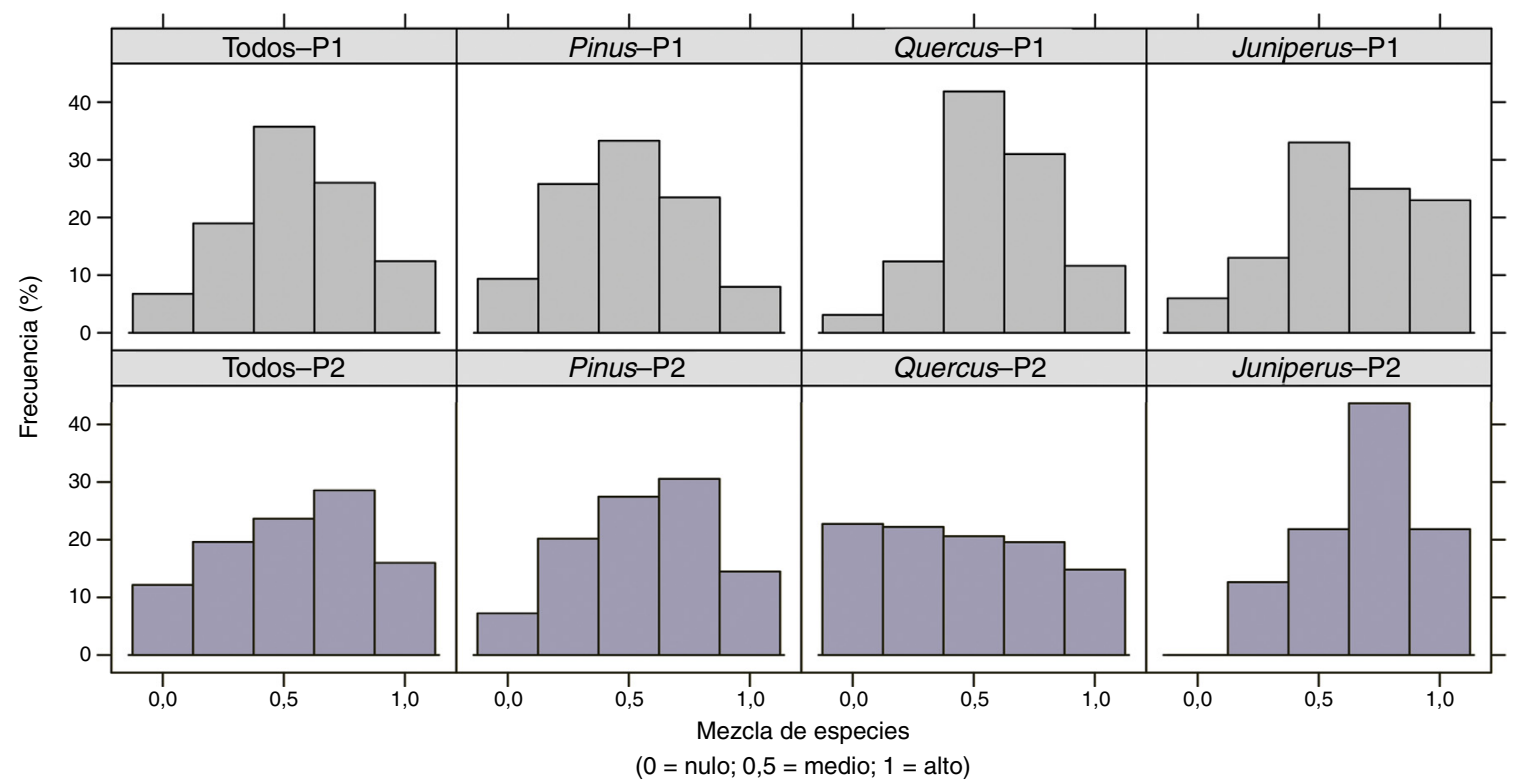

Figura 3. Grado de mezcla $\left(M_{i}\right)$ de especies por parcela y grupo en el área de estudio. P1: parcela 1; P2: parcela 2. Pinus, Quercus y Juniperus son los grupos de estudio; el grupo Todos incluye a todos los individuos y especies.

la categoría de diferenciación moderada (0.2-0.4), la categoría que le continúa es la clara (0.4-0.6) con poco más del 30\%, las otras categorías presentan menos de $10 \%$ de frecuencia. Para la P2 la distribución es muy similar, la mayoría se encuentra entre las categorías moderada y clara (fig. 4). La comparación entre parcelas no arrojó diferencias estadísticamente significativas.

En la figura 4 se pueden observar las distribuciones de las frecuencias en diferenciación diamétrica por grupo. Para el grupo Pinus, en P1, la categoría más representada es la moderada, y para P2, la clara, lo cual indica mayor diferenciación en P2. Para el grupo de los Quercus, se observa la categoría moderada con mayor frecuencia para las 2 parcelas, solo que en la P2 tiene mayor proporción; el grupo Juniperus, es muy similar en su distribución, también presentan en la categoría moderada la mayor frecuencia $(60 \%)$.

Índice de diferenciación dimensional de altura $\left(T H_{i}\right)$. En general, las distribuciones de alturas por parcela se muestran muy similares a lo encontrado en la diferenciación diamétrica, con una fuerte inclinación por la categoría moderada. En análisis por grupos, Pinus muestra una mayor tendencia, aunque ligera, a la diferenciación de alturas; por su parte los grupos Quercus y Juniperus tienen las mayores frecuencias en la categoría moderada, aunque muestran una ligera tendencia a la diferenciación en la P2. 


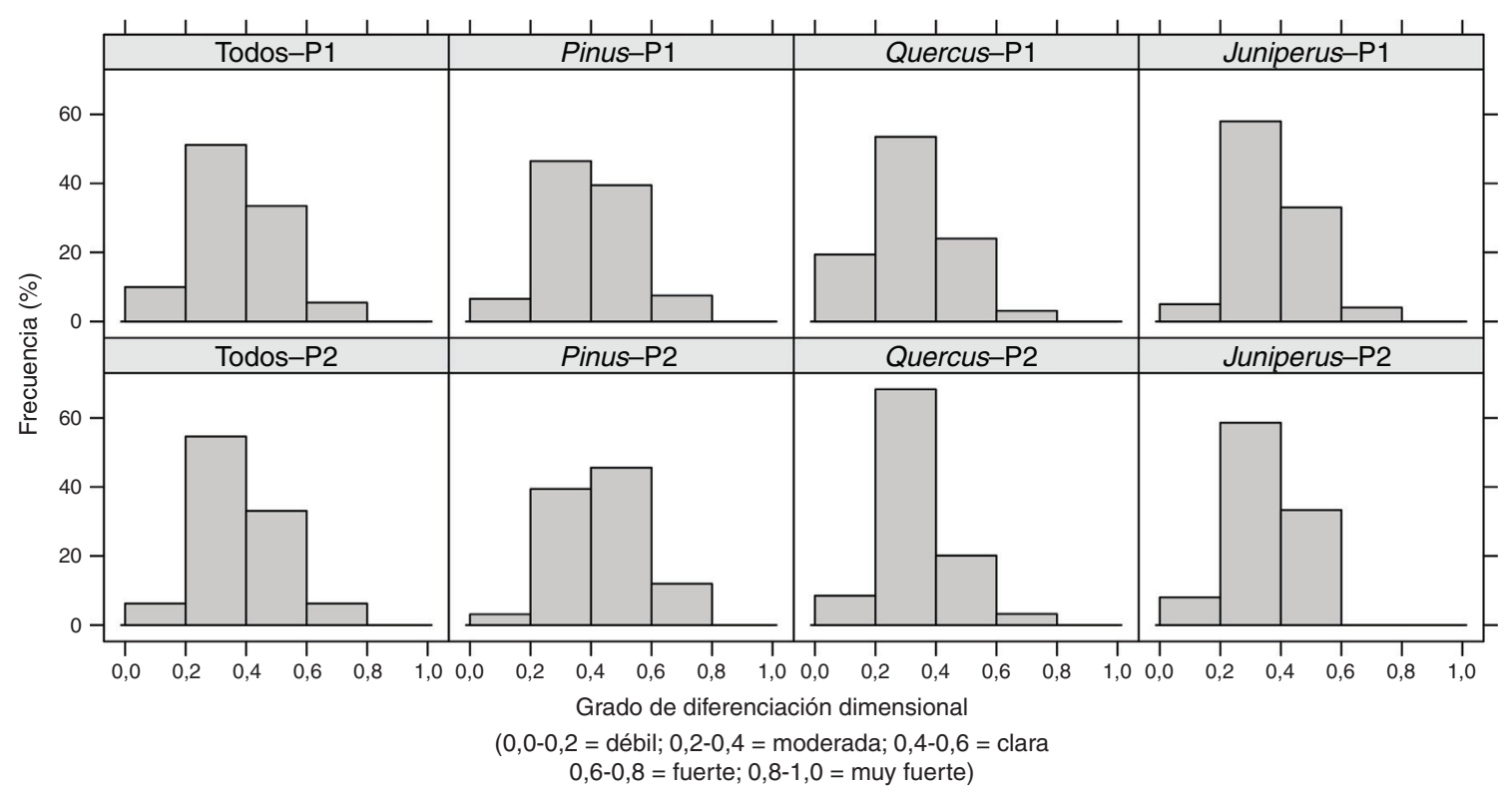

Figura 4. Grado de diferenciación diamétrica $\left(T D_{i}\right)$ por parcela y grupo en el área de estudio. P1: parcela 1; P2: parcela 2. Pinus, Quercus y Juniperus son los grupos de estudio; el grupo Todos incluye a todos los individuos y especies.

Índice de dominancia dimensional diamétrica $\left(U D_{i}\right)$. Las distribuciones de los valores de dominancia relativa no muestran ninguna tendencia en el análisis por parcela, pero en la clasificación de grupos se observan cambios en dicha distribución (fig. 5). El grupo Pinus tiene la mayor proporción en la categoría de 1, lo que indica que es una especie que domina sobre las que la rodean. Para el grupo de los Quercus, esta diferencia no es tan marcada, pero para los Juniperus la mayoría de las frecuencias caen en las categorías menores, más del $30 \%$ en la categoría 0 , indicando que es un género que en el área de estudio se encuentra rodeado por árboles más grandes, tanto en diámetro como en altura.

Índice de dominancia dimensional de altura $\left(U H_{i}\right)$. Las distribuciones de dominancia en altura son muy parecidas a las de diámetro, solo se pueden observar algunas diferencias en el análisis de grupos. En el caso de los Quercus, estos tienen las mayores frecuencias en las categorías menores en la P1, es decir, en su mayoría se encuentran rodeados por árboles más grandes, en tanto en la P2 la distribución se inclina más hacia la categoría de 0.50 . La comparación de medias entre parcelas $(\mathrm{P} 1=0.35 \pm 0.34 ; \mathrm{P} 2=0.45 \pm 0.32)$ muestra diferencias estadísticamente significativa ( $p=0.001 ; \mathrm{IC}=-0.168,-0.030)$.

\section{Discusión}

Los ecosistemas forestales son dinámicos ya que las perturbaciones son parte de sus procesos naturales y el motor de la sucesión (Pickett y White, 1985). De igual manera, el comportamiento determinado por los índices estructurales en las parcelas del Campus Ecológico Iturbide es resultado de un régimen de perturbaciones de incendios frecuentes y de baja severidad (Rubio-Camacho et al., 2016). Otros estudios en bosques templados donde se aplican las relaciones de vecindad, como los de Ávila et al. (2012) en bosques afectados por incendios con diferentes severidades, Castellanos et al. (2008), Castellanos, Treviño, Aguirre, Jiménez y Velázquez, 2010), Corral, Aguirre, Jiménez y Corral (2005) y Aguirre et al. (2003) en áreas afectadas por las intervenciones silvícolas, muestran también que los bosques presentan distribuciones aleatorias después de un disturbio o aprovechamiento silvícola.

Está ampliamente documentado que la estructura de los bosques mixtos de pino y encino está fuertemente influenciada por incendios forestales (Ávila et al., 2014; Rodríguez, 2008; Rodríguez y Fulé, 2003); cuando el fuego se presenta en los bosques, actúa como un agente regulador de la composición de especies, favoreciendo a unas y disminuyendo aquellas que no poseen adaptaciones que les ayuden a soportar el paso del fuego (Rodríguez, 1996; Rodríguez y Fulé, 2003).

En los ecosistemas mixtos de pino y encino, los pinos suelen conformar el estrato superior, mientras que los encinos se localizan en el estrato medio o sotobosque, siendo más tolerantes a la sombra (González-Tagle, 2005); no obstante, en nuestro estudio se encontró que los encinos exhiben mayores valores en la dominancia de alturas en la $\mathrm{P} 2$ con respecto a $\mathrm{P} 1$.

La importancia sobre el conocimiento generado a este respecto contribuye no solo al entendimiento de los procesos ecológicos de los ecosistemas de pino-encino, sino también al establecimiento de objetivos de manejo sustentable, adecuados a las condiciones locales. Los incendios frecuentes y de baja intensidad son recomendados para el mantenimiento y en algunos casos lograr la restauración mediante el uso del fuego en este tipo de ecosistemas (Rodríguez y Myers, 2010).

Para el análisis de la estructura y dinámica espacial en bosques mixtos resulta adecuado el desarrollo de análisis generalizados con todas las especies, y específicos por género o especie ya que brindan un panorama detallado sobre el comportamiento de bosque en general y de las especies (grupos) en particular, cuyos resultados se pueden asociar al estudio de perturbaciones. 


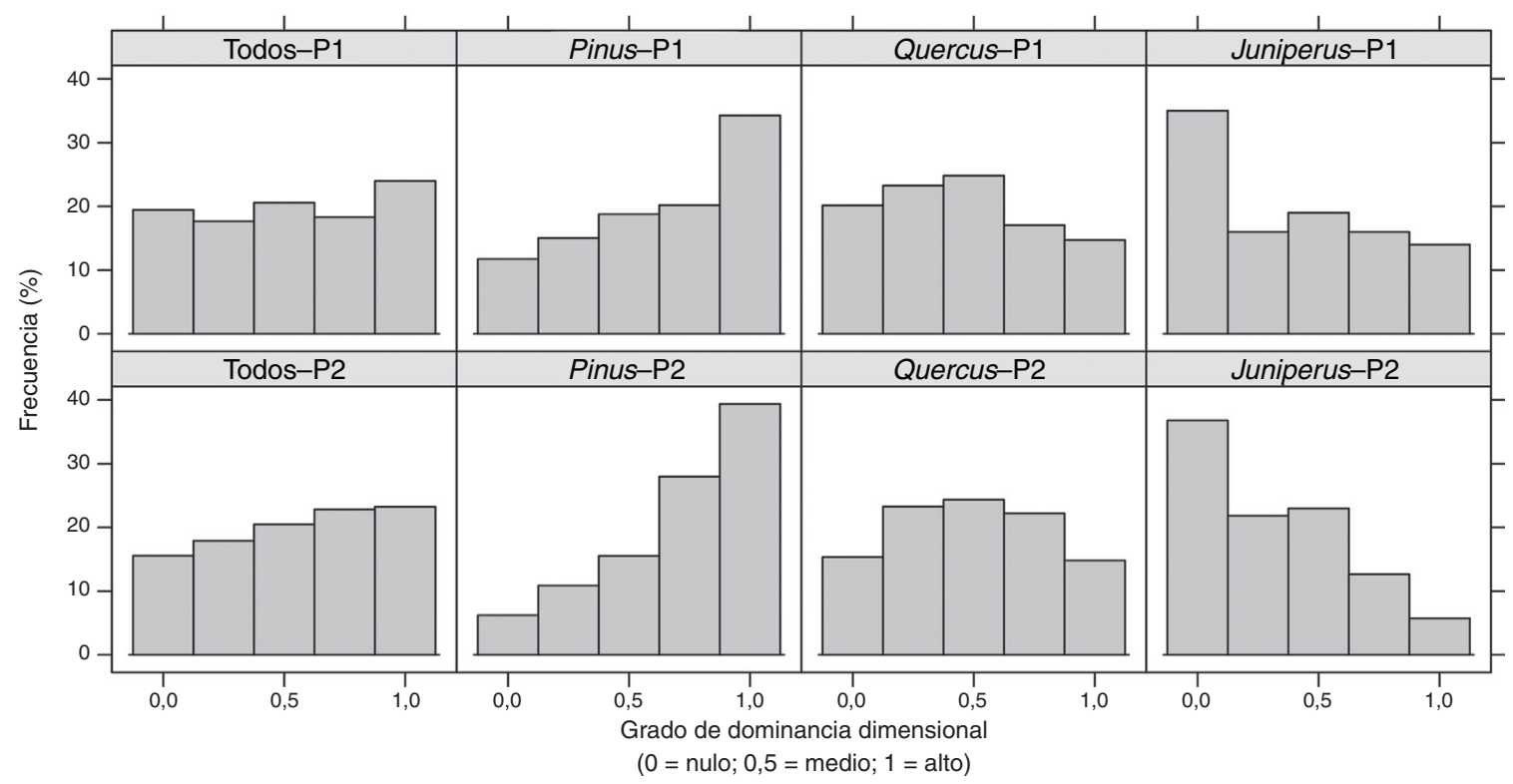

Figura 5. Grado de dominancia diamétrica $\left(U D_{i}\right)$ por parcela y grupo en el área de estudio. P1: parcela 1; P2: parcela 2. Pinus, Quercus y Juniperus son los grupos de estudio; el grupo Todos incluye a todos los individuos y especies.

\section{Referencias}

Agee, J. K. (1993). Fire ecology of Pacific Northwest forests. Washington, D.C: Island Press.

Aguirre, C. O. A. (2004). Índices para la caracterización del estrato arbóreo de ecosistemas forestales. Revista Ciencia Forestal en México, 27, 5-27.

Aguirre, C. O. A., Hui, G., von Gadow, K. y Jiménez, J. (2003). An analysis of spatial forest structure using neighborhood- based variables. Forest Ecology and Management, 183, 137-145.

Aguirre, C., Kramer, O. A. y Jiménez, H. J. (1998). Strukturuntersuchungen in einem Kiefern-Durchforstungsversuch Nordmexikos. Allgemeine Forst u. Jagdzeitung, 169, 213-220.

Albert, M. (1999). Analyse der eingriffsbedingten Strukturveränderung und Durchforstungsmodellierung in Mischbeständen. Alemania: Faculty of Forest Sciences, University Göttingen. Tesis doctoral.

Ávila, F. D. Y., González, T. M. A., Jiménez, P. J., Aguirre, C. O. A., Treviño, G. E. J. y Vargas J.L.B. (2012). Evaluación de la estructura espacial postincendio de rodales de Pinus hartwegii utilizando parámetros de vecindad en la Sierra Madre Oriental, México. Tropical and Subtropical Agroecosystems, 15, 377-387.

Ávila, F. D. Y., González, T. M. A., Jiménez, P. J., Aguirre, C. O. A., Treviño, G. E. J., Vargas, L. B., et al. (2014). Efecto de la severidad del fuego en las características de la estructura forestal en rodales de coníferas. Revista Chapingo Series Ciencias Forestales y del Ambiente, 20, 33-45.

Baddeley, A. y Turner, R. (2005). SPATSTAT: an R package for analyzing spatial point patterns. Journal of Statistical Software, 12, 1-42.

Balvanera, P., Cotler, H., Aburto, O. O., Aguilar, C. A., Aguilera, P. M. y Aluja, M. (2009). Estado y tendencias de los servicios ecosistémicos. En J. Sarukhán, R. Dirzo, R. González, y I. J. March (Eds.), Capital natural de México, vol. II: estado de conservación y tendencias de cambio (pp. 185-245). México, D.F: Conabio.

Castellanos, B. J. F., Treviño, G. E. J., Aguirre, C. O. A., Jiménez, P. J., Musalem, S. M. y López, A. R. (2008). Estructura de bosques de pino pátula bajo manejo en Ixtlán de Juárez, Oaxaca, México. Madera y Bosques, 14, 51-63.

Castellanos, B. J. F., Treviño, G. E. J., Aguirre, C. O. A., Jiménez, P. J. y Velázquez, V. M. A. (2010). Diversidad arbórea y estructura espacial de bosques de pino-encino en Ixtlán de Juárez, Oaxaca. Revista Mexicana de Ciencias Forestales, 1, 39-52.

Clark, P. J. y Evans, F. C. (1954). Distance to nearest neighbor as a measure of spatial relationships in populations. Ecology, 35, 445-453.
Corral, R. J. J., Aguirre, C. O. A., Jiménez, P. J. y Corral, R. S. (2005). Un análisis del efecto del aprovechamiento forestal sobre la diversidad estructural en el bosque mesófilo de montaña "El Cielo", Tamaulipas, México. Investigación Agraria: Sistemas y Recursos Forestales, 14, 217-228.

Fülder, K. (1995). Zur Strukturbeschreibung in Mischbeständen. Forstarchiv, $66,149-161$.

Gadow, K. V., Hui, G. Y. y Albert, M. (1998). Das Winkelmass - ein Strukturparameterzur Beschreibung der Individualverteilung in Waldbeständen. Centralblatt für das gesamte Forstwesen, 115, 1-9.

Gadow, K. V., Sánchez, S. O. y Álvarez, G. J. G. (2007). Estructura y crecimiento del bosque. Göetingen: Universidad de Göetingen.

Gadow, K. V., Zhang, C. Y., Wehenkel, C., Pommerening, A., Corral, R. J., Korol, M., et al. (2012). Forest structure and diversity. En T. Pukkala y K. V. Gadow (Eds.), Continuous cover forestry, managing forest ecosystems (pp. 23-55). Dordrecht, Países Bajos: Springer Science+Business Media B.V.

González-Tagle, M. A. (2005). Fire history and natural succession after forest fires in pine-oak forest. Alemania: Georg-August-Universität Göttingen. Tesis doctoral.

González-Tagle, M. A., Schwendemann, L., Jiménez, P. J. y Schulz, R. (2008) Forest structure and woody plant species composition along a fire chronosequence in mixed pine-oak forest in the Sierra Madre Oriental, Northeast Mexico. Forest Ecology and Management, 256, 161-167.

Himmelsbach, W., Treviño, G. E. J., González, R. H., González, T. M. A., Gómez, M. M. V., Aguirre, C. O. A., et al. (2011). Acclimatation of three cooccurring tree species to water stress and their role as site indicators in mixed pine-oak forests in the Sierra Madre Oriental, Mexico. European Journal of Forest Research, 131, 355-367.

Hui, G. Y. y Hu, Y. B. (2001). Measuring species spatial segregation in mixed forest. Forest Research, 14, 23-27.

Jardel-Peláez, E. J., Alvarado-Celestino, E., Morfín-Ríos, J. E., CastilloNavarro, F. y Flores-Garnica, J. G. (2009). Regímenes de fuego en ecosistemas forestales de México. En J. G. Flores-Garnica (Ed.), Impacto ambiental de incendios forestales (pp. 73-100). México, D.F: Mundi-Prensa.

Jiménez, P. J., Aguirre, O. A. C. y Kramer, H. (1998). Bestandestrukturanalyse im ungleichaltrigen Kiefern-Wacholder- Eichen-Mischwald Nordostenmexikos. Forstarchiv, 69, 227-234.

Mair, P., Wilcox, R. y Schoenbrodt, F. (2015). WRS2: a collection of robust statistical methods. Recuperado 23 Mar 2016 de: https://cran.rproject.org/web/packages/WRS2/index.html. 
Merganič, J., Merganičová, K., Marušák, R. y Audolenská, V. (2012). Plant diversity of forests. En A. Blanco y Y. H. Lo. (Eds.), Forest ecosystems-more than just trees (pp. 3-28) Croatia: InTech.

Pickett, S. T. A. y White, P. S. (1985). The ecology of natural disturbance and patch dynamics. New York: Academic Press.

Pommerening, A. (2002). Approaches to quantifying forest structures. Forestry, $75,305-324$.

Pommerening, A. (2008). Analyzing and modelling spatial woodland structure. Habilitations schrift. Vienna, Austria: University of Natural Resources and Applied Life Sciences. Tesis doctoral.

Pommerening, A. (2013). Woodland structure analysis and modelling Lab. Recuperado el 2 septiembre, 2013 de: http://www.crancod.org/wiki/ index.php?title=Main_Page\&redirect=no.

Pommerening, A. y Stoyan, D. (2006). Edge-correction needs in estimating indices of spatial forest structure. Canadian Journal of Forest Research, 36, 1723-1739.

Rodríguez, T. D. A. (1996). Incendios forestales. México, D.F: Mundi-Prensa.

Rodríguez, T. D. A. (2008). Fire regimes, fire ecology, and fire management in Mexico. Ambio: A Journal of the Human Environment, 37, 549-556.

Rodríguez, T. D. A. y Fulé, P. Z. (2003). Fire ecology of Mexican pines and fire management proposal. International Journal of Wildland Fire, 12, 23-37.
Rodríguez, T. D. A. y Myers, R. L. (2010). Using oak characteristics to guide fire regime restoration in Mexican pine-oak and oak forest. Ecological Restoration, 28, 304-323.

Rubio-Camacho, E. A., González, M. A., Alanís, R. E., Chávez-D, Á. A. y Aguirre-C, O. A. (2015). Analysis of the structure and diameter distribution in temperate forests under the perspective of the potential fire regime. Revista Chapingo Serie Ciencias Forestales y del Ambiente, 21, 28-294.

Rubio-Camacho, E. A., González, T. M. A., Benavides, S. J., Chávez, D. Á. A. y Xelhuantzi, C. J. (2016). Relación entre necromasa, composición de especies leñosas y posibles implicaciones del cambio climático en bosques templados. Revista Mexicana de Ciencias Agrícolas, 13, 2601-2614.

Sarkar, D. (2013). Lattice graphics. Package 'lattice'. Recuperado el 2 septiembre, 2013 de: http://cran.r-project.org/web/packages/lattice/lattice.pdf.

Sullivan, J. (2007). Juniperus flaccida. Fire effects information system. U.S. Department of Agriculture, Forest Service, Rocky Mountain Research Station, Fire Sciences Laboratory Recuperado el 2 septiembre, 2013 de: http://www.fs.fed.us/database/feis/plants/tree/junfla/introductory.html.

Whelan, R. J. (1995). The ecology of fire. Cambridge: Cambridge University Press.

Wilcox, R. R. (2005). Introduction to robust estimation and hypothesis testing. San Diego, CA: Elsevier Inc. 